\title{
Les syndicats britanniques face aux questions monétaires européennes
}

British trade unions and the european currency question 1988-2008

\section{Carine Berberi}

\section{CpenEdition}

\section{Journals}

Édition électronique

URL : http://journals.openedition.org/rfcb/1144

DOI : $10.4000 /$ rfcb. 1144

ISSN : 2429-4373

Éditeur

CRECIB - Centre de recherche et d'études en civilisation britannique

\section{Édition imprimée}

Date de publication : 27 juillet 2009

Pagination : 133-151

ISBN : 978-2-9115-8028-4

ISSN : 0248-9015

Référence électronique

Carine Berberi, « Les syndicats britanniques face aux questions monétaires européennes », Revue Française de Civilisation Britannique [En ligne], XV-2 | 2009, mis en ligne le 01 novembre 2016, consulté le 23 avril 2019. URL : http://journals.openedition.org/rfcb/1144 ; DOI : 10.4000/rfcb.1144

\section{(c) (†) $\ominus$}

Revue française de civilisation britannique est mis à disposition selon les termes de la licence Creative Commons Attribution - Pas d'Utilisation Commerciale - Pas de Modification 4.0 International. 


\title{
Les syndicats britanniques face aux questions monétaires européennes
}

\author{
Carole BERBÉRI \\ Université d'Orléans
}

\begin{abstract}
Alors que le mouvement syndical britannique avait été majoritairement hostile aux premiers projets européens de construction monétaire dans les années 1970 et 1980, il avait ensuite progressivement revu sa ligne de conduite et adopté un point de vue de plus en plus favorable à ces initiatives. À la fin des années 1990, le mouvement syndical soutenait désormais majoritairement l'Union Economique et Monétaire (UEM) et poussait les gouvernements britanniques à y adhérer. L'année 1988 avait été particulièrement déterminante puisqu'elle avait vu la conversion des syndicats aux projets européens à la suite du discours prononcé par Jacques Delors, président de la Commission Européenne, lors du Congrès du TUC de cette année-là. Toutefois, après plusieurs années de fort soutien à l'UEM et après avoir poussé les gouvernements de Tony Blair à adhérer à l'euro dès que possible, la majorité des syndicats britanniques ont de nouveau durci leur point de vue et semblent plus réticents à soutenir aujourd'hui une participation de la Grande-Bretagne à l'euro.
\end{abstract}

Comment pouvons-nous expliquer une ligne de conduite apparemment aussi inconstante ? Quelles raisons ont motivé ces changements d'attitude ? Dans la mesure où le mouvement syndical britannique est très vaste, nous chercherons à répondre à ces questions en examinant la ligne de conduite de la confédération syndicale britannique, le TUC (Trades Union Congress), mais aussi des principaux syndicats qui lui étaient affiliés au cours des années 1990 et 2000. Nous nous concentrerons sur cinq syndicats : l'AEEU $^{1}$ (Amalgamated Engineering and Electrical Union), le $\mathrm{GMB}^{2}$ (General Municipal and Boilermakers' Union), $\mathrm{MSF}^{3}$

\footnotetext{
${ }^{1}$ L'AEEU a vu le jour en 1992 à la suite du regroupement de deux syndicats de métier, l'AEU (Amalgamated Engineering Union) et l'EETPU (Electrical, Electronic, Telecommunication and Plumbing Union) qui avaient déjà connu eux-mêmes plusieurs transformations. L'AUEW (Amalgamated Union of Engineering Workers) avait par exemple adopté le sigle AEU à la suite du départ de TASS (Technical, Administrative and Supervisory Section) et de la réorganisation de ses trois sections (Engineering, Foundry, Construction) en 1986.

${ }^{2}$ Le GMB a été créé en 1924 sous le nom de GMWU (General and Municipal Workers Union) à la suite de la fusion de plusieurs syndicats. Il subit de nombreux regroupements tout au long de son histoire, notamment avec le syndicat des chaudronniers (Boilermakers' Union) en 1982 ou celui des employés de bureau APEX (Association of Professional, Executive, Clerical and Computer Staff) en 1989. GMB ne devint le sigle officiel du syndicat qu'en 1989.

${ }^{3}$ MSF est issu de la fusion en 1988 de l'ASTMS (Association of Scientific, Technical and Managerial Staffs) et de TASS, deux syndicats professionnels. Au cours des années 1970,
} 
(Manufacturing, Science and Finance Union), le TGWU ${ }^{4}$ (Transport and General Workers' Union) et UNISON ${ }^{5}$.

\section{Les syndicats, majoritairement hostiles aux premiers projets européens de construction monétaire (1970-83)}

$\mathrm{Au}$ cours des années 1970, la majeure partie du mouvement syndical britannique, que ce soit au niveau du TUC ou des principaux syndicats qui lui étaient affiliés, regardait d'un mauvais œil les premiers projets européens de construction monétaire. En ce qui concernait le TUC, il avait d'abord pris peu au sérieux le plan Werner ${ }^{6}$, estimant que le projet d'union monétaire était peu crédible et non réalisable ${ }^{7}$. Pour autant, il n'avait pas refusé d'y réfléchir, parvenant dès cette époque à en saisir les principales implications économiques et politiques. Il avait ainsi souligné divers dangers suscités par ce projet. Premièrement, au sein d'une union monétaire, il serait plus difficile de parvenir au plein emploi dans la mesure où la mobilité interne serait relativement limitée et où la suppression des taux de change impliquerait le recours à d'autres mesures comme les subventions internes ou les investissements étrangers. Deuxièmement, il serait plus difficile d'assurer la compétitivité de la Grande-Bretagne puisque l'on ne pourrait plus dévaluer la livre pour rendre les exportations plus attractives. Troisièmement, ce type d'union aurait des conséquences sur la souveraineté britannique puisqu'il impliquerait un État fédéral unifié ${ }^{8}$. Petit à petit, cette attitude critique évolua vers une ligne de conduite plus précise et plus hostile en raison notamment du contexte économique peu favorable. À la fin des années 1970, le Conseil général du TUC était clairement opposé à tout projet d'union monétaire, considérant qu'il ne permettrait pas aux pays européens de répondre plus facilement à la récession économique à laquelle le monde devait faire face, et ce d'autant moins qu'il limiterait leur capacité à agir au niveau national.

Le TUC avait également adopté une ligne de conduite très critique à l'égard du Mécanisme des Taux de Change (MTC) du Système Monétaire Européen (SME) même s'il n'avait formulé aucune objection fondamentale à son encontre. En fait, il estimait que l'adoption d'un régime de changes fixes dominé par le deutschemark présentait des dangers pour les régions européennes les plus pauvres et les pays à monnaie faible s'il ne prévoyait pas un mécanisme d'ajustement approprié ainsi

TASS avait fait partie de la section des cols blancs de l'AUEW mais il s'en était détaché ensuite en mai 1986.

${ }^{4}$ Notons que depuis 2007, le TGWU a fusionné avec AMICUS, qui résultait lui-même de la fusion de MSF et de l'AEEU en 2002, pour former le syndicat UNITE.

${ }^{5}$ UNISON résulte de la fusion en juillet 1993 de trois syndicats du secteur public NALGO (National and Local Government Officers Association), NUPE (National Union of Public Employees) et COHSE (Confederation of Health Service Employees), et recrute actuellement à la fois dans les secteurs public et privé.

${ }^{6}$ Ce plan, présenté en octobre 1970, est considéré comme le véritable point de départ de l'UEM.

${ }^{7}$ Amy VERDUN, European Responses to Globalization and Financial Market Integration. Perceptions of Economic and Monetary Union in Britain, France and Germany, Basingstoke: Macmillan, 2000, p. 125.

${ }^{8}$ TUC, TUC Report 6-10 September 1971, p. 319. 
qu'un transfert de ressources au niveau communautaire ${ }^{9}$. En 1978, il fit donc dépendre une éventuelle participation de la Grande-Bretagne au MTC du SME de diverses conditions similaires à celles définies par le Parti travailliste à l'époque. Ces conditions étaient au nombre de six, le système devait: (1) favoriser la croissance économique dans l'économie mondiale, (2) imposer des obligations équivalentes aux pays à monnaie faible et forte, (3) se montrer viable sur le long terme, (4) faire preuve de flexibilité en acceptant les ajustements, (5) comporter un transfert de ressources des pays les plus riches vers les plus pauvres, (6) ne pas nuire au dollar ni au Système Monétaire International ${ }^{10}$. Dans la mesure où ces conditions avaient peu de chances d'être satisfaites, nous pouvons nous demander si l'attitude du TUC n'était pas plus hostile au mécanisme des taux de change qu'il ne le laissait paraître.

Cette ligne de conduite était majoritairement partagée par les principaux syndicats britanniques qui avaient rejeté dès le départ les projets de construction monétaire, en raison essentiellement de la perte de pouvoir économique et de souveraineté qu'ils impliquaient pour les États. Ainsi, la plupart des syndicats que nous avons choisi d'étudier estimaient que le projet d'union monétaire allait nuire à la souveraineté des institutions britanniques et entraînerait une perte d'autonomie importante pour la Grande-Bretagne en matière économique, qui l'empêcherait de résoudre les problèmes propres au pays ${ }^{11}$. Leur hostilité au MTC du SME s'expliquait par ces préoccupations politiques mais aussi par des raisons économiques, semblables à celles évoquées par le TUC. Le TGWU et l'ASTMS, notamment, mirent en évidence que le MTC imposerait des règles trop strictes pour des pays faibles économiquement et fragiliserait leur économie et leur industrie ${ }^{12}$. Entre 1970 et 1979, seuls le GMWU et APEX étaient favorables aux initiatives européennes d'intégration monétaire, soutenant la perspective d'une union monétaire ainsi que le MTC du SME. Alors que le GMWU craignait que le refus de participer au mécanisme des taux de change ne réduise l'influence du Royaume-Uni et ne l'empêche de peser véritablement sur les futures décisions européennes, APEX voulait, grâce au MTC, assurer le maintien d'un taux de change approprié pour la livre afin de ne pas répéter des crises semblables à celle de $1931^{13}$.

Ce fut entre 1979 et 1983 que le mouvement syndical britannique se montra particulièrement uni sur les questions monétaires européennes, une très grande majorité s'y opposant sous l'emprise de la gauche et de l'AES. Même le GMWU avait modifié son point de vue. Il faut dire que la majorité des syndicats soutenaient alors l'adoption d'une stratégie économique alternative (Alternative Economic Strategy - AES) qui impliquait un État très centralisé puisqu'elle avait pour éléments majeurs une politique d'expansion cherchant à restaurer le plein emploi, la multiplication des nationalisations, la planification économique, le contrôle de

${ }^{9}$ TUC, TUC Report 3-7 September 1979, p. 297.

${ }^{10}$ Ibid.

${ }^{11}$ Pour le TGWU, voir par exemple: TGWU Our Wider Aims, January 1979, p. 11-12; Minutes and Records of the Proceedings of the $28^{\text {th }}$ Biennial Delegate Conference, 9-13 July 1979 , p. 33.

12 'Joint “no" to Britain joining the EMS', ASTMS Journal, Nov./Dec. 1978, p. 1.

${ }^{13}$ Interview de John Edmonds, 21-28 janvier 2003. 
l'inflation et l'imposition de contrôles sur le commerce international. Dans la mesure où cette stratégie était incompatible à bien des égards avec le traité de Rome $^{14}$, la majorité des syndicats réclamèrent le retrait de la Grande-Bretagne de la Communauté Economique Européenne (CEE), ce qui impliquait le rejet des projets de construction monétaire.

À cette époque, seuls NALGO et COHSE affichèrent un point de vue plus nuancé car ils commençaient à prendre conscience de l'importance de la CEE, de la nécessité de s'impliquer davantage dans les institutions européennes, et de coopérer avec les syndicats européens. S'ils favorisaient l'AES comme les autres syndicats, ils soutenaient en même temps la $\mathrm{CEE}^{15}$. La direction du TUC devait également être mise à part car le Conseil général du TUC refusait de faire campagne en faveur du retrait de la Grande-Bretagne de la CEE, préférant critiquer certains aspects de la Communauté $^{16}$. En fait, son attitude était très incohérente car tout en soutenant l'AES, le Conseil général du TUC préconisait une adhésion de la Grande-Bretagne au MTC du SME afin de parvenir à une plus grande stabilité monétaire face à l'absence de coopération internationale dans ce domaine ${ }^{17}$. Cette ligne de conduite était paradoxale mais avant-gardiste puisqu'alors le Parti travailliste n'était pas favorable au mécanisme des taux de change.

\section{Une période de remise en cause et de mutisme (1983-88)}

$\mathrm{Au}$ cours de cette période, les initiatives monétaires européennes, et les initiatives européennes de manière générale, furent peu abordées par les syndicats britanniques, les dirigeants syndicaux cherchant à éviter tout débat sur l'Europe et sur les questions monétaires afin de laisser une marge de manœuvre suffisante au Parti travailliste qui tentait d'évoluer sur ces questions.

Effectivement, le mouvement travailliste tout entier traversait alors un processus de modernisation à la suite de la défaite électorale de 1983. En ce qui concernait le mouvement syndical, il tenta de modifier ses politiques de façon à répondre davantage aux attentes des Britanniques - électeurs et syndiqués -, et d'attirer davantage de membres, ses effectifs ainsi que le taux de syndicalisation ayant beaucoup baissé depuis 1979. Cette prise de conscience qu'il fallait moderniser le mouvement syndical encouragea une amorce de changement envers l'Europe, mais l'évolution progressive vers un point de vue plus favorable se traduisit, tout d'abord, par une période de silence : les questions européennes furent très peu abordées entre 1983 et 1987. Par un accord accepté pratiquement par tous les syndicats, aucune résolution ne fut plus proposée sur cette question lors des congrès du TUC pour les raisons suivantes: aucun des congrès des principaux syndicats n'avait alors opéré de volte-face sur l'Europe, et si un vote avait été

${ }^{14}$ Effectivement, l'AES reposait sur une économie planifiée alors que traité de Rome reposait sur la philosophie de libre-échange.

${ }^{15}$ COHSE, 1980 Conference - Final Agenda, p. 68-69.

'NALGO must look after members' interests in EEC', Public Service, March 1981, Vol. 55, $\mathrm{N}^{\circ} 3$, p. 7.

${ }^{16}$ TUC, TUC Report 6-10 September 1982, p. 260-261.

${ }^{17}$ TUC, The Battle for Jobs, TUC Economic Review 1983, February 1983, p. 44. 
organisé, il aurait débouché sur une attitude hostile. La direction du TUC préféra donc éviter tout débat pour laisser une marge de manœuvre au Parti travailliste ${ }^{18}$. Elle ne se désintéressa pas de l'Europe pour autant: sous l'impulsion de Norman Willis ${ }^{19}$, la confédération se tourna lentement vers une attitude plus positive à l'égard de l'Europe. Il est toutefois difficile d'étudier son évolution sur les questions monétaires européennes car ces dernières n'apparurent jamais dans les débats, ni dans les publications du TUC de l'époque. Nous pouvons supposer que la confédération y était favorable, d'autant plus qu'elle cherchait alors un taux de change plus concurrentiel pour la livre.

Comme le TUC, la plupart des syndicats que nous avons choisi d'étudier mentionnèrent très peu les questions monétaires au cours de cette période. Ils étaient certainement toujours opposés à ces questions dans la mesure où ils continuaient à soutenir l'AES qui impliquait le retrait de la Grande-Bretagne de la CEE et donc le rejet des projets européens d'intégration monétaire. Certains syndicats commencèrent tout de même à évoluer sous la pression de leur direction : le GMWU et l'AUEW abandonnèrent progressivement leur politique de retrait de la CEE mais sans adopter une ligne de conduite ferme vis-à-vis de l'Europe, et encore moins visà-vis du MTC du SME, en raison de l'hostilité persistante de la base. APEX, quant à lui, continua à soutenir le maintien de la Grande-Bretagne au sein de la CEE et sans doute également le MTC du SME ${ }^{20}$.

\section{Une amorce de changement (1988-1992)}

À partir de 1988-1989, le mouvement syndical britannique commença véritablement à évoluer sur les questions européennes et monétaires, et à adopter une ligne de conduite de plus en plus favorable à leur égard en raison de divers facteurs.

Premièrement, le mouvement syndical avait dû revoir ses croyances en matière de politique économique après l'abandon de l'AES. Au cours des années 1980, le mouvement syndical avait pris conscience qu'il était désormais impossible pour un gouvernement de mener une politique économique autonome. Mises à part les critiques formulées par certains membres de la gauche contre l'AES, deux exemples lui avaient particulièrement servi de leçon : celui du gouvernement français de François Mitterrand qui n'avait pas réussi à mener un programme national expansionniste, très proche de l'AES, au tout début des années 1980, et l'exemple des États-Unis qui en une seule décennie avaient perdu leur place de premier créditeur mondial pour occuper celle de premier débiteur mondial en poursuivant des politiques économiques de manière indépendante ${ }^{21}$. À partir de la fin des années

${ }^{18}$ Lewis MINKIN, The Contentious Alliance, Trade Unions and the Labour Party, Edinburgh: Edinburgh U. P., 1991, p. 455-456.

${ }^{19}$ Norman Willis, qui était personnellement favorable à l'intégration européenne, était devenu le nouveau Secrétaire général du TUC en 1984.

${ }^{20}$ Carine BERBERI, Le Parti travailliste et les syndicats face aux questions monétaires européennes, Paris : L'Harmattan, 2005, p. 258-259.

${ }^{21}$ Denis MacSHANE, French Lessons for Labour, London: Fabian Society, 1986, p. 5. 
1980, le mouvement syndical commença donc à envisager de nouvelles stratégies économiques et notamment des stratégies économiques européennes prenant en compte le MTC du SME et l'UEM : «Une nouvelle approche dans les années 1990 devra refléter le nouveau contexte économique et surtout l'influence des initiatives européennes. Il sera tout simplement impossible de mener, avec succès, des politiques économiques sur le long terme, indépendamment du processus visant à créer une Union économique et monétaire en Europe $»^{22}$.

Deuxièmement, les syndicats britanniques souhaitaient réagir aux nombreuses lois anti-syndicales qui avaient été votées par les gouvernements conservateurs de Margaret Thatcher entre 1979 et 1990. Au début des années 1990, les syndicats étaient devenus des organismes très réglementés et leur pouvoir avait été considérablement réduit alors même que la liberté des patrons britanniques avait été fortement accrue. Qui plus est, une grande flexibilité avait été introduite dans la législation sur le travail et l'on avait assisté à une baisse des prestations sociales. Les syndicats décidèrent donc de se tourner vers l'Europe afin d'offrir de meilleures conditions de travail et de vie aux Britanniques et de regagner l'influence qu'ils avaient perdue au niveau national, perspective tout à fait envisageable puisque la législation européenne ne pouvait être modifiée ou supprimée par un gouvernement conservateur :

Une campagne coordonnée pour la mise en æuvre de la dimension sociale pourrait sans aucun doute restaurer certains des droits syndicaux dont le gouvernement nous a privés ces neuf dernières années. Il est temps pour les Européens peu enthousiastes de reconsidérer la CEE et de prendre conscience que, même si elle n'est pas parfaite en tous points, elle offre le premier espoir tangible pour améliorer les droits des travailleurs et les dispositions sociales en Grande-Bretagne depuis $1979 .^{23}$

Les syndicats britanniques s'intéressèrent d'autant plus aux projets européens, et notamment aux initiatives européennes en matière sociale et d'emploi, que ces dernières se multiplièrent au cours des années 1980 et 1990. Après que l'Acte unique européen leur eut donné une nouvelle impulsion avec l'adoption de la Charte sociale en 1989 et la promotion du dialogue social, le traité de Maastricht accéléra le processus d'un progrès en parallèle de l'Europe économique et de l'Europe sociale.

Denis MacSHANE, 'British Unions and Europe' in Ben PIMLOTT \& Chris COOK (eds.) Trade Unions in British Politics: the First 250 Years, New York: Longman, 1991, p. 289.

22 'A new approach in the 1990 s will have to reflect changed circumstances, above all the influence of European developments. It will, quite simply, be impossible in the long run to successfully conduct economic policy in isolation from the move towards greater economic and monetary union within Europe'. TUC, Towards 2000: Managing the Economy, August 1990, p. 7.

23 'A concerted campaign for the social dimension to be implemented could well restore some of the trade union rights that the Government has denied us over the last nine years. It is time for reluctant Europeans to look again at the EEC and realise that, while it is far from perfect in every respect, it offers the first tangible hope for the improvement of workers rights and social provisions in Britain since 1979'. Paula FLEMING, 'Europe 1992: An opportunity for UK industry', AEU Journal, September 1988, p. 14. 
Tous ces projets suscitèrent l'intérêt des syndicats, et ce d'autant plus que les gouvernements conservateurs y étaient opposés.

Quatrièmement, le mouvement syndical britannique fut encouragé à revoir sa ligne de conduite vis-à-vis des projets monétaires européens en raison de l'évolution du Parti travailliste sur ces questions. En 1990 le parti avait totalement modifié son point de vue : il soutenait désormais officiellement une participation de la GrandeBretagne au MTC du SME si plusieurs conditions étaient satisfaites ${ }^{24}$, et favorisait le projet d'UEM même si celui-ci était soumis à diverses réserves relatives, en particulier, à un fonctionnement plus démocratique de la Banque Centrale Européenne (BCE), à une convergence économique suffisante entre les pays membres, ou à l'adoption de politiques régionales efficaces ${ }^{25}$.

\section{Le TUC : un soutien progressif au système monétaire européen}

L'évolution du TUC sur les questions européennes fut visible dès son Congrès de 1988. À cette occasion, Jacques Delors, président de la Commission de Bruxelles, prononça un discours qui fut accueilli avec enthousiasme par les syndicalistes. Le président de la Commission énonça effectivement des idées qui correspondaient à leurs objectifs et idéaux : un rôle dans l'élaboration des politiques économiques et sociales, une meilleure protection des droits de leurs membres et un accroissement des droits pour les groupes en difficulté, comme les chômeurs. Grâce à Delors, les syndicats découvraient la possibilité de regagner l'influence qu'ils avaient perdue au niveau national en agissant au niveau européen ${ }^{26}$.

Lors de ce congrès, le Conseil général du TUC présenta également Maximising the Benefits, Minimising the Costs, document évaluant les implications de l'Acte unique européen. Il y soulignait l'importance du marché unique pour la GrandeBretagne et les syndicats britanniques, tout en insistant sur un développement en parallèle de la dimension économique et de la dimension sociale ${ }^{27}$. Enfin, ce Congrès vit l'adoption, sans dissension, d'une résolution définissant une stratégie européenne pour les syndicats, qui rompaient ainsi avec plusieurs années de silence forcé $^{28}$. À partir de ce moment-là, le TUC adopta véritablement une attitude plus constructive sur les questions européennes et accrut considérablement son travail sur l'intégration européenne. L'Europe n'était plus perçue comme une menace, mais comme une occasion à saisir pour améliorer les conditions de travail et de vie des Britanniques.

Le TUC évolua également sur les questions monétaires européennes même s'il fit preuve de davantage de prudence. Ainsi, le MTC du SME ne fut pas mentionné

\footnotetext{
${ }^{24}$ Quatre conditions devaient être réunies : un taux de change efficace pour la livre, des arrangements appropriés entre banques centrales, une bonne politique régionale au niveau européen, et des politiques économiques communautaires visant à promouvoir la croissance.

${ }^{25}$ NEC, Economic and Monetary Union, 6 November 1990, pp. 2-7.

${ }^{26}$ Stephen GEORGE, Ben ROSAMOND, 'The European Community' in Martin J. SMITH, Joanna SPEAR (eds.) The Changing Labour Party, London: Routledge, 1992, p. 179.

${ }^{27}$ TUC, Maximising the Benefits, Minimising the Costs, August 1988, p. 9-10, pp. 28-29.

${ }^{28}$ TUC, TUC Report 5-9 September 1988, pp. 669-671.
} 
lors du Congrès de 1988. Il fallut attendre l'année suivante, après que le sommet de Madrid eut adopté le rapport Delors ${ }^{29}$ en juin 1989, pour que le Conseil général décide de réexaminer cette question. Son point de vue fut ensuite défini dans divers documents : Europe 1992. Progress Report on Trade Union Objectives (1989), 1990 Budget Submission (1990) et Towards 2000: Managing the Economy (1990). Le Conseil général du TUC y indiquait qu'il soutenait une participation de la GrandeBretagne au MTC du SME. Selon lui, ce mécanisme pouvait avoir trois principaux avantages : il favoriserait le commerce britannique en supprimant l'incertitude liée à la fluctuation des taux de change ; il faciliterait la baisse des taux d'intérêt puisque le gouvernement chercherait sur le long terme à respecter le taux d'inflation moyen de la CEE; il protégerait la livre des attaques spéculatives ${ }^{30}$. La participation de la Grande-Bretagne au MTC du SME était toutefois soumise à plusieurs réserves. Tout d'abord, elle devait être accompagnée de politiques visant à accroître la coordination macroéconomique et à réduire les disparités régionales au sein de la $\mathrm{CEE}^{31}$. Afin d'aider les exportateurs britanniques la Grande-Bretagne devait également négocier une entrée à des marges de fluctuation élargies de plus ou moins $6 \%$ ainsi qu'un taux de change s'élevant à 2,70 DM environ ${ }^{32}$.

En dépit de cette attitude favorable, le TUC fut assez indifférent à l'entrée de la livre dans le MTC du SME en octobre 1990 : il ne montra aucun enthousiasme mais il ne s'opposa pas non plus à cette décision bien qu'aucune de ses conditions n'ait été satisfaite. Il émit simplement quelques critiques à l'encontre du gouvernement conservateur, lui reprochant notamment d'avoir adhéré à un taux de change trop élevé, et alors même que le taux d'inflation de la Grande-Bretagne était beaucoup plus fort que celui des autres pays européens. Cette indifférence était certainement motivée par le fait que les syndicats affiliés au TUC faisaient encore preuve de scepticisme envers le mécanisme des taux de change et que le Parti travailliste avait été très embarrassé par la décision du gouvernement conservateur.

Le TUC avait également adopté une attitude plus favorable à l'égard de l'UEM, la volte-face fut même encore plus frappante ici puisque le TUC y était auparavant totalement opposé. Son changement d'attitude apparut en 1989, le Conseil général indiquant dans Europe 1992. Progress Report on Trade Union Objectives qu'il accueillait favorablement le rapport Delors qui pouvait être bénéfique aux travailleurs britanniques. Diverses réserves étaient encore une fois émises. Le Conseil général regrettait notamment la trop grande importance qui avait été accordée à l'union monétaire au détriment de l'union économique, et plus précisément à la lutte contre l'inflation plutôt qu'à la poursuite du plein emploi, ainsi que le fonctionnement peu démocratique du Système Européen des Banques Centrales, susceptible d'agir hors de tout contrôle politique ${ }^{33}$. La principale préoccupation du Conseil général à l'époque semblait néanmoins être les répercussions de l'UEM sur la compétitivité de l'industrie britannique, et sur les

\footnotetext{
${ }^{29}$ Se situant dans le prolongement du rapport Werner, ce rapport proposait des étapes concrètes pour parvenir à l'UEM.

${ }^{30}$ TUC, 1990 Budget Submission, January 1990, p. 14-15.

${ }^{31}$ TUC, Europe 1992. Progress Report on Trade Union Objectives, September 1989, p. 13.

32 TUC, Towards 2000: Managing the Economy, August 1990, p. 8-9.

${ }^{33}$ TUC, Europe 1992. Progress Report on Trade Union Objectives, op. cit., p. 12.
} 
négociations salariales. Le Conseil général redoutait effectivement que les patrons hésitent davantage à augmenter les salaires au sein d'une UEM puisqu'ils ne pourraient plus compenser une perte de compétitivité par une baisse du taux de change. Malgré ces réserves et préoccupations, le point de vue du TUC était encore peu défini. En fait, le TUC se contentait surtout de répéter les conclusions des travaux de la Confédération européenne des syndicats (CES), prenant en compte les remarques de la CES, mais ne cherchant pas à pousser sa réflexion plus avant ${ }^{34}$.

Comme la confédération syndicale, la majorité des syndicats que nous avons choisi d'étudier s'orientèrent vers une ligne de conduite plus positive et plus ferme à l'égard de l'Europe à la fin des années 1980, encouragés dans cette direction par le discours prononcé par Jacques Delors au congrès du TUC de 1988. Pour certains, comme pour le GMWU, l'AEU et APEX, ce changement s'opéra assez rapidement. Cherchant à obtenir de nouveaux avantages sociaux grâce à l'Europe, ils insistèrent dès cette époque sur une mise en place harmonieuse du marché unique et sur une dimension sociale européenne. Pour d'autres, comme le TGWU ou MSF, l'évolution fut plus timide, voire difficile, encouragée essentiellement par la direction compte tenu de l'anti-européanisme persistant au sein de ces syndicats. Au début des années 1990, la majorité des syndicats avaient néanmoins pris conscience de l'importance et des implications des initiatives européennes.

Les syndicats s'intéressèrent également de plus en plus aux questions monétaires, comme l'indiqua un responsable du TGWU : « Notre changement de politique [sur les questions monétaires européennes] a cö̈ncidé avec 'l'offensive de charme' menée par Jacques Delors contre les syndicats traditionnellement opposés à l'Europe et avec le point de vue plus favorable adopté par le Parti travailliste à l'égard de l'Europe sous la direction de Kinnock ${ }^{35}$.

En ce qui concernait le MTC du SME, les principaux syndicats (GMB, AEU, TGWU) y étaient maintenant devenus favorables, estimant que le mécanisme pouvait être profitable à l'économie britannique en luttant contre l'inflation, en réduisant la spéculation monétaire et en facilitant les prises de décision à long terme pour les entreprises grâce à la stabilité des changes. Le GMB et l'AEU soulignaient toutefois que la participation au MTC du SME priverait les industries exportatrices de la possibilité de dévaluer la livre en cas de difficultés ${ }^{36}$. Les autres syndicats, et notamment MSF et NALGO, demeuraient plus réservés en raison de la perte de souveraineté et de contrôle des instruments de gestion économique que le MTC du SME imposerait aux États membres ${ }^{37}$.

${ }^{34}$ TUC, Towards 2000: Managing the Economy, op. cit., p. 7.

35 'Our policy change [on European monetary questions] coincided with the Jacques Delors "charm offensive" against traditionally Eurosceptic trade unions and the more positive approach to EC membership of the Kinnock-led Labour Party'. Entretien avec un responsable du TGWU, 1-2 avril 2003.

36 'ECUS, EMUS, ERMS - The European Monetary Jungle', AEU Journal, August 1991, p. 21.

GMB \& UCW, A New Agenda. Bargaining for Prosperity in the 1990s, 1990, pp. 3-4.

TUC, TUC Report 3-7 September 1990, p. 566.

${ }^{37}$ NALGO, The Economy - A NALGO Review, May 1991, p. 53.

MSF, Europe 1992 - What about the workers?, 1988, p. 7. 
L’adhésion de la Grande-Bretagne au MTC du SME en octobre 1990 suscita des réactions relativement identiques parmi ces syndicats puisque tous critiquèrent les conditions d'adhésion, et notamment le taux surévalué de la livre. Pour pallier ce problème, les syndicats recommandèrent essentiellement un réalignement des monnaies au sein du SME, et plus précisément une dévaluation de la livre ${ }^{38}$.

Dans un premier temps, et de manière similaire au TUC, les syndicats affichèrent des lignes de conduite peu déterminées sur la question de l'union monétaire. L'AEU et le GMB furent les syndicats qui se caractérisèrent par l'attitude la plus positive, soutenant ce projet même s'ils critiquaient la trop grande indépendance de la BCE et les répercussions que l'union monétaire pourrait avoir sur les négociations salariales. Le GMB avait la politique la plus réfléchie et la plus constructive des deux. Selon lui, l'union monétaire était susceptible d'apporter les avantages suivants : suppression des coûts de transaction, disparition de l'incertitude liée à la variation des taux de changes, harmonisation des taux d'intérêt, comparaison plus facile des niveaux de vie et des coûts de production au sein de la Communauté, suppression de la domination de la Bundesbank ${ }^{39}$. L'AEU et le GMB considéraient également que la participation à l'UEM permettrait à la GrandeBretagne de ne pas s'isoler et de pouvoir influencer la mise en place de l'union monétaire $^{40}$. Les autres syndicats, comme MSF et NALGO, restèrent plus nuancés, exprimant de plus en plus des préoccupations relatives à la baisse des salaires, à la perte de pouvoir et aux inégalités régionales que l'union monétaire pourrait provoquer, mais sans avoir de point de vue encore bien défini, ce qui témoignait de leur manque de réflexion ${ }^{41}$. Le TGWU avait, lui, une ligne de conduite un peu plus constructive et positive, comme l'illustra un article rédigé par Bill Morris et publié dans Tribune en décembre 1990. Bill Morris y remettait notamment en question l'idée que l'UEM occasionnerait une baisse des salaires, estimant que ces derniers ne déterminaient pas à eux seuls la compétitivité des entreprises. De même, il résolvait le problème lié à la perte de pouvoir dans le domaine monétaire en insistant sur un certain nombre de conditions dont devait dépendre une participation de la Grande-Bretagne à l'UEM : le fonctionnement des institutions européennes devrait être plus démocratique, la mise en place de l'UEM ne devrait pas nuire aux personnes le plus en difficulté, la convergence économique devrait être accompagnée d'une convergence sociale ${ }^{42}$.

\section{La prise de conscience des enjeux de l'Union Economique et Monétaire (1992-1997)}

Ce fut dans le cadre des débats sur le traité de Maastricht que les syndicats britanniques furent contraints de préciser leur point de vue sur les projets européens

\footnotetext{
${ }^{38}$ L'AEU fit toutefois preuve d'une plus grande réserve et se montra assez silencieux sans doute afin de ne pas embarrasser les travaillistes.

${ }^{39}$ GMB, 'Europe - Meeting the Challenge', Special CEC Report to Congress'91, 1991, pp. $15-16$.

${ }^{40}$ Ibid. , p. 17.

${ }^{41}$ MSF, Europe: The Way Ahead, An MSF Discussion Document, June 1991, pp. 8-10.

NALGO, The Economy - A NALGO Review, op. cit., pp. 54-55.

${ }^{42}$ Bill MORRIS, 'Unions and the new Europe', Tribune, 7 December 1990, p. 6.
} 
de construction monétaire. Le TUC décida de soutenir le traité malgré ses imperfections $^{43}$ et de s'opposer aux deux clauses d'exemption négociées par le gouvernement conservateur, qu'il s'agisse de celle sur le volet social ou de celle sur l'UEM. Selon le Conseil Général, la clause d'exemption sur l'UEM risquait d'isoler la Grande-Bretagne au sein de la CE : non seulement le Royaume-Uni ne pourrait plus influencer la mise en place de l'union monétaire mais il ne pourrait également plus faire pression pour que la $\mathrm{BCE}$ soit localisée à Londres ${ }^{44}$. Deux principales raisons motivaient le soutien du TUC à l'UEM. D'une part, cette dernière imposerait de toute façon des critères de convergence similaires aux objectifs économiques du gouvernement Major. D'autre part, elle permettrait de mettre un terme à la domination de la Bundesbank en Europe ${ }^{45}$. Ce soutien n'était toutefois pas inconditionnel puisque le TUC continuait à insister sur l'importance de la croissance et de l'emploi afin d'assurer une mise en place harmonieuse de l'UEM.

La majorité des syndicats que nous avons choisi d'étudier précisèrent également leur ligne de conduite à l'égard de l'union monétaire : la plupart d'entre eux (notamment le TGWU, NALGO, MSF et même le GMB et l'AEEU) décidèrent de soutenir la clause d'exemption que le gouvernement Major avait négociée sur l'UEM, adoptant ainsi une politique radicalement différente de celle du TUC. Il faut dire que la majorité des syndicats étaient alors préoccupés par les critères de convergence $^{46}$ qui ne répondaient pas à leurs attentes en raison de leur caractère purement monétariste : regrettant que ces critères ne prennent pas davantage en compte l'emploi et la croissance, les syndicats redoutaient les répercussions qu'ils pourraient avoir sur l'économie européenne, et ce d'autant plus que les pays européens devaient alors faire face à la récession économique et à une montée du chômage $^{47}$. Les syndicats s'inquiétaient également de la perte de contrôle et de souveraineté que l'UEM engendrerait pour la Grande-Bretagne, critiquant notamment le fonctionnement peu démocratique de la BCE et le pouvoir excessif donné aux banquiers ${ }^{48}$. Notons qu'en règle générale, la base de ces syndicats affichait une opposition beaucoup plus farouche à l'UEM et au traité de Maastricht que la direction.

Parmi les syndicats retenus pour notre étude, NALGO était indubitablement le plus véhément puisqu'il choisit de rejeter en bloc le traité de Maastricht et la ligne de conduite du TUC à son égard. Il estimait désormais que la ratification du traité entraînerait une perte de souveraineté, une hausse du chômage, une baisse des salaires et une réduction des dépenses destinées aux services publics, la GrandeBretagne ne pouvant plus dévaluer sa monnaie pour rester compétitive. En tant que représentant du secteur public, NALGO s'inquiétait surtout des conséquences que le

\footnotetext{
${ }^{43}$ Le TUC adopta ainsi la même ligne de conduite que le Parti travailliste.

44 TUC, Unions after Maastricht, February 1992, p. 25.

${ }^{45}$ TUC, TUC Report 7-11 September 1992, p. 139.

${ }^{46}$ Les critères de convergence venaient effectivement d'être déterminés dans le cadre de la préparation du traité. Les syndicats s'inquiétaient essentiellement des critères relatifs aux finances publiques qui impliquaient la réduction des dépenses allouées aux services publics et empêchaient l'adoption de toute politique économique et sociale à caractère socialiste.

${ }^{47}$ AEEU, 1993 Conferences, p. 120. Interview de John Edmonds, 21-28 janvier 2003.

${ }^{48}$ MSF, Annual Conference 7-11 May 1994, p. 1.
} 
critère de convergence relatif au déficit public (3\% du PIB) pourrait avoir sur l'économie britannique : le déficit britannique s'élevant déjà à $5 \%$, il soulignait que le respect de ce critère impliquerait des réductions massives des dépenses publiques $^{49}$. Bon nombre de membres de ce syndicat partageaient ces inquiétudes.

Après la création de UNISON, à la suite de la fusion entre NALGO, COHSE et NUPE en 1993, le syndicat conserva un point de vue hostile à l'égard du traité. La direction du syndicat était assez nuancée dans la mesure où elle reconnaissait l'importance des initiatives européennes de manière générale, notamment celles dans le domaine social (sans doute afin de concilier le point de vue de chaque syndicat) tout en critiquant les critères de convergence et les limites fixées par le traité concernant les dépenses publiques ${ }^{50}$. Plusieurs sections locales étaient néanmoins plus véhémentes, demandant au TUC de cesser de soutenir le traité et de s'opposer à toute participation de la Grande-Bretagne à la troisième phase de l'UEM $^{51}$.

COHSE était alors le seul syndicat parmi ceux que nous avons choisi d'étudier à adopter un point de vue plus favorable à l'UEM. Ce syndicat avait effectivement pris conscience que l'autonomie de la Grande-Bretagne en matière de politique monétaire était très limitée, cette dernière étant fortement influencée par les décisions de la Bundesbank et il regrettait donc la clause d'exemption sur l'UEM. Selon Tony Chandler, l'un de ses responsables, l'UEM aurait pour avantage de permettre à chaque pays membre de pouvoir influencer la politique économique européenne et de faire économiser des millions grâce à la suppression des coûts de transaction $^{52}$.

\section{Des préoccupations communes : l'emploi et la croissance}

À partir du début des années 1990, le mouvement syndical britannique se concentra de plus en plus sur la lutte contre le chômage aux niveaux national et européen. Il était non seulement préoccupé par les difficultés économiques auxquelles la Grande-Bretagne et les pays européens étaient confrontés ${ }^{53}$, mais aussi par les critères de convergence, dont le caractère trop monétariste pouvait engendrer une hausse du nombre de chômeurs. Le TUC et tous les syndicats retenus pour notre étude préconisèrent donc des actions visant à créer des emplois au niveau national, l'élaboration de stratégies coordonnées de lutte contre le chômage au niveau européen (le TUC proposa notamment un programme destiné à assurer la croissance, l'emploi et la compétitivité du domaine industriel en collaboration avec la CES) et ils insistèrent sur l'application des nouvelles initiatives européennes en matière

${ }^{49}$ Astrid STUBBS, 'Brussels sprouts forlorn hope’, NALGO News Daily, N²8, 9 September 1992, p. 3.

50 'National Conference 1995 Final Agenda', UNISON FOCUS, 28April 1995, p. 8.

51 'Union First Conference Preliminary Agenda', UNISON WEEK, 18 February 1994, p. 5.

${ }^{52}$ Tony CHANDLER, 'Maastricht at a glance', COHSE Bulletin, June 1993, p. 6.

${ }^{53}$ Les pays européens devaient alors faire face à un contexte économique et politique difficile en raison de la récession économique et des conflits dans l'ex-Yougoslavie. Qui plus est, la Grande-Bretagne était confrontée à un ralentissement de la croissance et à une hausse du chômage. 
sociale et d'emploi ${ }^{54}$. Alors que certains syndicats, comme le TGWU, MSF, le GMB ou l'AEEU, se concentrèrent, au niveau national, sur les difficultés de l'industrie manufacturière britannique, d'autres, comme UNISON, prêtèrent davantage attention au secteur public, secteur où étaient employés la majorité de ses membres.

La priorité accordée à la création d'emplois et à la croissance ne fut pas sans répercussions sur la politique suivie par le mouvement syndical britannique à l'égard de l'UEM. Ainsi, les syndicats demandèrent que les objectifs de croissance et d'emploi soient pris en compte dans le processus de mise en place de l'UEM et recommandèrent une redéfinition des critères de convergence ${ }^{55}$. Ils souhaitaient que ces derniers ne répondent plus seulement à des objectifs purement financiers mais prennent en compte les données réelles des économies, donc la croissance et l'emploi. En témoigne la motion suivante à laquelle le GMB accorda son soutien lors du congrès du TUC de 1993: «Le Congrès estime que les objectifs de croissance et d'emploi ainsi que la coopération économique doivent être le fondement de la convergence économique et qu'ils doivent apporter la crédibilité définitive aux dispositions de mise en place de la nouvelle monnaie ${ }^{56}$.

En raison de ce contexte, certains syndicats renforcèrent leur opposition à l'UEM durant ces années. La direction du TGWU devint progressivement plus réservée, voire alarmiste sur cette question. À partir de 1996, Bill Morris soulignait par exemple que l'UEM pourrait avoir des conséquences catastrophiques sur les emplois britanniques, citant l'exemple de la participation au MTC du SME qui, selon lui, avait provoqué une hausse du chômage en Grande-Bretagne ${ }^{57}$. La direction de UNISON devint également franchement hostile au projet d'union monétaire, estimant que le respect des critères de convergence, et particulièrement de ceux relatifs aux finances publiques, engendrerait des réductions des dépenses et des compressions des effectifs dans le secteur public ${ }^{58}$. Ces craintes étaient partagées par la majorité des membres du syndicat.

Les autres syndicats (le GMB, l'AEEU et MSF), comme la majorité du mouvement syndical, décidèrent eux de favoriser l'UEM. Notons que la direction de MSF avait progressivement adopté un point de vue plus favorable à l'UEM au fur et à mesure que ce projet se concrétisait. Elle semblait désormais admettre que la monnaie unique permettrait de mettre un terme à la spéculation monétaire et qu'il était dangereux pour la Grande-Bretagne de ne pas y participer dans la mesure où la

\footnotetext{
${ }^{54}$ Les syndicats accueillirent ainsi favorablement l'initiative d'élaborer en 1993 un Livre Blanc sur la croissance, la compétitivité et l'emploi, ainsi que les propositions du gouvernement suédois concernant l'inclusion dans le traité de Maastricht d'un chapitre sur l'emploi et l'idée d'un Pacte Européen de Confiance pour l'Emploi en 1996.

${ }^{55}$ TUC, The Next Phase in Europe, August 1993, p. 7.

TUC, TUC Report 6-10 September 1993, pp. 506-507.

TGWU, Preliminary Agenda, 35th Biennial Delegate Conference 5-9 July 1993, p. 13.

56 'Congress believes that growth and employment targets, and economic co-operation, must be the basis for economic convergence and provide the ultimate credibility of new currency arrangements'. TUC, TUC Report 6-10 September 1993, p. 506.

${ }_{57}$ Bill MORRIS, 'Jobs on the line', The Guardian, 9 September 1996, p. 13.

${ }^{58}$ UNISON, 'Decisions of the Third Annual UNISON Delegate Conference', UNISON FOCUS Online, Issue 34.
} 
mondialisation de l'économie rendait impossible la poursuite de toute politique économique nationale ${ }^{59}$. Cette évolution était néanmoins mal accueillie par quelques-unes de ses sections locales qui demeuraient très sceptiques envers $l^{\prime}$ Europe et $l^{\prime} U_{E M}{ }^{60}$. Le soutien de ces syndicats était soumis à diverses réserves puisque comme nous l'avons déjà noté, ils demandaient une redéfinition des critères de convergence et insistaient pour que l'UEM soit accompagnée d'une croissance économique, d'une relance des emplois et d'une véritable dimension sociale.

Quant au TUC, alors sous la direction de John Monks, fervent pro-Européen, il adopta une ligne de conduite de plus en plus favorable à l'UEM, à tel point qu'il devint le premier corps constitué important à adopter un point de vue enthousiaste sur cette question au Royaume-Uni ${ }^{61}$. Ce point de vue résultait de la crainte de voir la Grande-Bretagne exclue d'un centre de décision majeur, de la volonté de profiter des avancées européennes dans le domaine social, mais aussi d'une réflexion sur les avantages et inconvénients propres à l'UEM. Après que le sommet de Madrid de 1995 eut fixé le $1^{\text {er }}$ janvier 1999 comme date de lancement de la monnaie unique, le TUC avait effectivement décidé de suivre une politique plus constructive à l'égard de l'UEM et de réfléchir davantage à ses enjeux. Début 1997, le TUC n'opposait même plus avantages et inconvénients de l'UEM dans ses publications, mais avantages d'une adhésion et inconvénients d'une non-adhésion, ce qui illustrait sa politique très favorable à l'euro. Il indiquait également désormais que la GrandeBretagne devrait adhérer à la monnaie unique dès le $1^{\text {er }}$ janvier 1999 , avec la première vague de participants ${ }^{62}$.

\section{Les syndicats face à l'euro : un rôle de fer de lance? (1997-2002)}

Le TUC ne modifia pas son attitude à la suite de la victoire des travaillistes en mai 1997, et continua à encourager le nouveau gouvernement de Tony Blair à adhérer rapidement à l'euro, et ce d'autant plus que la livre était surévaluée et qu'elle nuisait aux entreprises exportatrices. En dépit de cet enthousiasme, le Congrès du TUC de 1997 se montra très prudent, voire réservé, à l'égard des projets européens de construction monétaire. Mis à part le rapport du Conseil général sur l'Europe, aucune résolution ne fut adoptée sur cette question et toutes les motions proposées furent mises de côté. Lors de son intervention, John Edmonds, parlant au nom du Conseil général, expliqua qu'il était difficile pour le TUC d'adopter un point de vue bien défini compte tenu des nombreuses incertitudes qui existaient sur l'UEM :

Enfin, il y a l'énigme de l'UEM. D'ici le prochain Congrès, le gouvernement britannique devra prendre une décision officielle sur la participation à la monnaie unique. Chacun s'attend à ce que la Grande-Bretagne reste en dehors, mais les termes du débat ont

\footnotetext{
${ }^{59}$ Elle soutint ainsi un article de Stuart HOLLAND qui tenait ce type de discours. (Beyond Maastricht: a new strategy for jobs and recovery in Europe, London: MSF, May 1993).

${ }^{60}$ MSF, 'MSF and Europe', At Work, October 1993, p. 2.

${ }^{61}$ LABOUR RESEARCH DEPARTMENT, 'Delegates vote for EU Monetary Union', Labour Research, October 1996, p. 3.

${ }^{62}$ TUC, 'TUC calls for Task Force on EMU', Eurobriefing, N5, February 1997, p. 3.
} 
beaucoup évolué et il se pourrait qu'ils évoluent encore. La date du lancement semble toujours être janvier 1999 mais, le directeur de la Bundesbank ayant lui-même confirmé qu'il ne serait pas catastrophique de la différer, il n'est pas impossible d'envisager janvier 2001. ${ }^{63}$

Fin 1997, le TUC était parvenu à déterminer une ligne de conduite précise qui prenait en compte les éléments de mise en place de la monnaie unique. Après avoir été fortement déçue, en octobre 1997, par la décision des travaillistes de ne pas adhérer à l'UEM avant les prochaines élections ${ }^{64}$, la confédération reprit son rôle de fer de lance sur l'euro, critiquant la politique trop prudente du gouvernement et l'incitant à être plus positif, plus constructif. La Grande-Bretagne devait non seulement adhérer à l'euro pour aider les industries exportatrices britanniques, mais aussi pour influencer les événements en Europe. Le TUC craignait effectivement que la Grande-Bretagne ne se trouve désormais isolée au sein de l'UE et de la zone euro, et ne soit exclue des décisions économiques importantes ${ }^{65}$.

Afin d'assurer une adhésion rapide de la Grande-Bretagne à l'euro, le TUC déploya beaucoup d'énergie pour intensifier le débat sur l'UE en Grande-Bretagne. Il organisa ainsi des séminaires régionaux, des conférences, et publia diverses brochures pour que les syndicalistes, les entreprises locales et le secteur public prennent conscience des implications de l'UEM et des préparatifs nécessaires. Il était même prêt à aider le gouvernement travailliste à convaincre les Britanniques du bien-fondé d'une adhésion à l'euro: «L'histoire risque de se répéter si l'on s'abstient indéfiniment d'adhérer à l'euro. Je veux que l'on s'attaque aux eurosceptiques. Je veux que le Congrès contribue à créer le climat qui encouragera le gouvernement à aller de l'avant ${ }^{66}$. «Il est essentiel que les syndicats britanniques prennent la tête et jouent un rôle constructif dans une campagne en faveur de l'euro ${ }^{67}$.

Le soutien du TUC à la monnaie unique n'était pas inconditionnel pour autant.

\footnotetext{
63 'Lastly, of course, there is the enigma of EMU. Before next Congress the British government will have to make a formal decision whether to join the single currency. Everyone expects Britain to stay outside, but the terms of the debate have shifted sharply and they may shift again. January 1999 still looks like the probable start date, but, with even the President of the German Bundesbank confirming that a delay would not be disastrous, January 2001 is still an outside possibility'. TUC, Verbatim Reports, September 1997.

${ }^{64}$ Le gouvernement avait défini cinq conditions qui devaient être respectées pour que la Grande-Bretagne puisse adhérer à l'euro. Ces conditions portaient sur la convergence économique, la flexibilité, les répercussions sur les investissements, les services financiers ainsi que sur la croissance et l'emploi.

${ }^{65}$ TUC, 'EMU "commitment to join is welcome but delay is disappointing", 27 October 1997.

TUC, ' 1999 will be the year of the Euro', 31 December 1998.

66 'An indefinite abstention from the euro risks history repeating itself. I want us to take on the euro-sceptics. I want Congress to help create the conditions which will encourage the government to go forward'. TUC, Congress Report, September 1999.

67 'It is essential that UK trade unions take a lead and play a full part in a campaign to join the euro'. TUC, Congress Report, September 2000.
} 
Premièrement, la confédération continuait à insister sur le fait que la mise en place de l'UEM devait être accompagnée de progrès en matière sociale et d'emploi afin d'assurer le succès de l'UEM mais aussi d'obtenir l'adhésion des Britanniques. À partir de 1999, le TUC alla même jusqu'à indiquer qu'il ne ferait campagne pour le « oui » dans un référendum que si le gouvernement s'engageait davantage en faveur de la dimension sociale. Deuxièmement, le TUC critiquait toujours la BCE, qu'il s'agisse de son fonctionnement peu démocratique ou de ses attributions axées essentiellement sur la lutte contre l'inflation. Troisièmement, il s'opposait aux critères de convergence, trop stricts et trop monétaires.

L'AEEU, le GMB et MSF étaient également devenus progressivement de plus en plus enthousiastes à l'égard de l'UEM, se rapprochant de la ligne de conduite du TUC. Après l'arrivée des travaillistes au pouvoir en mai 1997, ils continuèrent, dans un premier temps, à se caractériser par une attitude favorable mais prudente, estimant qu'il valait mieux ne pas adhérer à l'euro si ce n'était pas dans l'intérêt du pays. Ils accueillirent donc favorablement les cinq conditions définies par le gouvernement de Tony Blair pour adhérer à l'euro le 27 octobre 1997. Toutefois, à partir de 1998, l'AEEU et le GMB (aussi bien la direction que la base) atténuèrent leurs réserves et adoptèrent un point de vue de plus en plus enthousiaste, encourageant le gouvernement à y adhérer dès que possible, et ce pour diverses raisons. Premièrement (et cette raison était primordiale) une participation à l'euro aiderait les industriels britanniques alors confrontés aux problèmes liés à la surévaluation de la livre et aux taux d'intérêt trop élevés. Deuxièmement, elle mettrait un terme au climat d'incertitude entourant l'attitude de la GrandeBretagne sur cette question, climat qui nuisait aux emplois britanniques en dissuadant les multinationales d'investir dans le pays ${ }^{68}$. Troisièmement, elle améliorerait l'image du Royaume-Uni tout en renforçant son influence et sa crédibilité au sein de l'UE. Quatrièmement, la monnaie unique apporterait de nombreux avantages, parmi lesquels : stabilité des taux de change, suppression des coûts de transaction, maintien de l'influence du Royaume-Uni en Europe, taux d'intérêt plus faibles et comparaison plus facile des prix et des salaires. ${ }^{69}$

Le MSF, pour sa part, continua à faire dépendre son soutien à l'UEM d'un certain nombre de conditions, insistant notamment sur la mise en place lente et progressive de l'euro afin d'assurer la convergence économique des États membres. Il fallut attendre 2002, soit après sa fusion avec l'AEEU, pour qu'elles disparaissent progressivement. En 2002, il poussait ainsi le gouvernement à annoncer rapidement une date pour le référendum afin de mettre un terme aux incertitudes et d'aider ainsi l'industrie manufacturière qui souffrait de la surévaluation de la livre ${ }^{70}$. Certaines de ses sections locales, minoritaires, restèrent toutefois opposées à la monnaie unique, critiquant la $\mathrm{BCE}$ et les critères de convergence susceptibles d'entraîner des réductions des dépenses publiques. Par ailleurs, d'autres reprochèrent à la direction l'absence de politique claire sur la participation ou non à la monnaie unique et demandèrent l'élaboration d'une brochure précise sur cette question, ce qui

68 'Supporting UK manufacturing', Union News, N`35, September 2002, p. 14-15.

AEEU, The European Single Currency. A Guide for AEEU Members, October 1999, p. 7.

${ }^{69}$ AEEU, The European Single Currency. A Guide for AEEU Members, op. cit., p. 5.

70 'End of the Euro poll "hokey cokey" - says Amicus', 9 June 2002. 
témoignait d'un manque de débat interne sur l'euro ${ }^{71}$.

L'AEEU et le GMB étaient donc les deux syndicats les plus enthousiastes. Comme le TUC, ils déployèrent beaucoup d'énergie pour stimuler un débat sérieux sur l'euro en Grande-Bretagne. L'AEEU fut notamment très actif, cherchant à jouer par l'intermédiaire de Ken Jackson, un rôle de fer de lance sur la monnaie unique au sein du mouvement syndical. Dès 1997, Ken Jackson encouragea ainsi les syndicats à s'engager davantage dans le débat sur l'euro et à informer les Britanniques des répercussions de l'UEM : «En tant que syndicats qui ont ouvert la voie en 1988, nous devrions, une fois encore, avoir le courage d'ouvrir la voie sur la monnaie unique. Nous devrions dissiper l'ignorance et les doutes injustifiés que beaucoup de personnes ont sur la monnaie unique. Outre le fait qu'ils soient injustifiés, ces doutes sont souvent des convictions dangereuses $»^{72}$.

En ce qui concernait le TGWU et UNISON, la direction et la majorité de leurs syndiqués, conservèrent des lignes de conduite beaucoup plus réservées puisqu'ils n'étaient pas prêts à soutenir une participation de la Grande-Bretagne à l'UEM tant que certaines conditions n'étaient pas satisfaites. Non seulement ils insistaient sur le respect des cinq tests définis par le gouvernement travailliste, mais ils voulaient également que des progrès soient faits dans le domaine social et de l'emploi, et que le secteur public ne soit pas menacé, comme l'indiqua clairement Dave Prentis, Secrétaire général de UNISON : «Nous soutenons les conditions établies par le gouvernement, mais nous resterons prudents tant que personne ne pourra nous assurer que l'UEM ne menace ni les emplois, ni l'économie, ni nos services publics; ces garanties sont cruciales pour nous ${ }^{73}$. À partir de 1999-2000, UNISON et le TGWU lièrent de plus en plus l'UEM aux services publics, souhaitant que l'euro ne nuise pas à leur maintien et à leur modernisation. UNISON, en tant que principal représentant de ce secteur, était le plus alarmiste, affirmant que la satisfaction des critères de convergence pouvait entraîner de fortes réductions des dépenses publiques et risquait d'accroître les privatisations ${ }^{74}$. En 1997, son Congrès demanda, par conséquent, que des mesures compensatoires soient adoptées pour pallier les effets déflationnistes provoqués par le respect des critères et il insista pour que les critères de convergence soient redéfinis pour assurer la croissance et l'emploi. Outre l'insistance sur la protection du service public et l'importance d'un renforcement de la dimension sociale européenne, le TGWU et UNISON réclamaient que la $\mathrm{BCE}$ ait un fonctionnement plus transparent et plus démocratique, et que ses attributions ne soient seulement pas limitées à la stabilité des prix, mais promeuvent la croissance et l'emploi au sein de la zone euro.

\footnotetext{
${ }^{71}$ AMICUS (MSF Section), Annual Conference Agenda 8-11 June 2002, p. 18.

72 'As trade unions who led the way in 1988, we should have the courage once again to lead the way on single currency. We should dispel the ignorance and ill-founded doubts that many people have on the single currency. These doubts are often more than just ill-founded; they are dangerous beliefs'. TUC, Verbatim Reports, 1997.

73 'We support the government's tests but, crucially for us, until anyone can give us a guarantee that jobs are safe, the economy is safe, and our public services are safe, we will remain cautious'. Dave PRENTIS, 'Euro: Crunch Time', Fusion Magazine, Feb-March 2001, p. 22.

${ }^{74}$ UNISON, UNISON Speech at TUC EMU Conference, May 1999.
} 
En dépit de leurs réserves, le TGWU et UNISON cherchèrent, comme l'AEEU et le GMB, à stimuler l'organisation d'un débat sérieux et constructif sur la monnaie unique en Grande-Bretagne afin d'en déterminer les enjeux et d'informer les Britanniques. En 1997, au Congrès du TUC, Bill Morris, recommanda, par exemple, que le processus d'une éventuelle participation de la Grande-Bretagne soit ralenti en raison du manque d'information sur l'euro :

\begin{abstract}
Laissez-moi préciser mon point de vue. On ne demande pas de stopper le train [européen en direction de l'UEM]; on demande seulement qu'il soit ralenti pour pouvoir distinguer tout au moins où nous allons. Nous voulons étudier les signaux qui sont tout à fait clairs. Nous voyons des pertes d'emploi, des réductions des dépenses publiques, des réductions dans les budgets destinés aux services publics. Nous voulons que le plein emploi soit notre destination. Ce que je veux vous dire aujourd'hui est très simple : s'il vous plaît ralentissez. Organisons d'abord un débat sur l'UEM puis prenons une décision. ${ }^{75}$
\end{abstract}

\title{
Des lignes de conduite plus réservées (2002-2008)
}

Depuis 2002, le point de vue de ces syndicats s'est un peu durci en raison notamment de l'élection de nouveaux dirigeants, tels Derek Simpson à la tête d'AMICUS pour la section représentant l'AEEU ou Paul Kenny en ce qui concerne le GMB, qui ont accru l'influence de la gauche eurosceptique au sein du mouvement syndical. Il faut également noter que la probabilité d'une adhésion de la GrandeBretagne à l'euro est devenue de plus en plus faible au fil du temps, notamment après que les travaillistes eurent à nouveau repoussé cette perspective à la suite de l'évaluation des cinq tests, le 9 juin 2003. À cette occasion, Gordon Brown avait indiqué clairement que la Grande-Bretagne ne pouvait toujours pas adhérer à la monnaie unique, une seule des cinq conditions (celle concernant le secteur financier) étant respectée. De manière étonnante, cette ligne de conduite suscita peu de controverses au sein du mouvement syndical puisque la plupart des syndicats auxquels nous nous sommes intéressés se montrèrent favorables au report d'une adhésion. GMB fut le seul syndicat à se montrer critique, reprochant au gouvernement sa timidité qui mettait en danger les emplois.

Le climat plus tendu et l'hostilité croissante des syndicats opposés à la monnaie unique, comme UNISON et le TGWU, se sont manifestés clairement lors des Congrès du TUC de 2002 et de 2003. À ces deux occasions, le Conseil général du TUC a été contraint de modérer son enthousiasme afin de parvenir à un consensus sur la question de l'euro. Les résolutions adoptées lors de ces Congrès ont ainsi fait dépendre la participation de la Grande-Bretagne d'un certain nombre de conditions. Alors que le Congrès de 2002 a insisté sur le respect des cinq critères

\footnotetext{
75 'Let me make it absolutely clear. We are not arguing to stop the train; we are asking for it to be slowed down so that we can at least read the route map. We want to read the signals which are loud and clear. We see job losses, public expenditure reductions, and public service cuts. We want our destination to be full employment. My message today is a very simple one: please slow down. Let us have the debate and then decide. TUC, Verbatim Reports', 10 September 1997.
} 
définis par Gordon Brown, un taux de change concurrentiel entre la livre et l'euro, un engagement plus fort du gouvernement en faveur de la dimension sociale européenne et des garanties concernant les répercussions d'une adhésion sur les dépenses publiques, celui de 2003 a recommandé le respect des cinq critères, la réforme du Pacte de stabilité et la mise en place par le gouvernement d'initiatives européennes en matière sociale. Par ailleurs, une faible majorité de syndicats $(52,7 \%)$ ont soutenu le rapport du Conseil général qui affichait une ligne de conduite en faveur de l'euro en 2003.

\section{Conclusion}

Après que les syndicats eurent été majoritairement hostiles aux projets monétaires européens en raison de leur soutien à l'AES au début des années1980, ils évoluèrent progressivement, et plus ou moins rapidement, vers une ligne de conduite plus favorable, stimulés par leur direction et par celle du TUC. À partir de 1988, les initiatives européennes dans le domaine social les encouragèrent à revoir leur point de vue, et ce d'autant plus que leur pouvoir avait été considérablement réduit au niveau national par les lois anti-syndicales des gouvernements conservateurs, et qu'ils avaient pris conscience qu'il était désormais impossible de mener des politiques autonomes. Le mouvement syndical britannique se tourna donc vers l'Europe et vers ses projets monétaires pour tenter de regagner l'influence qu'ils avaient perdue au niveau national et améliorer ainsi les conditions de travail et de vie des Britanniques.

Compte tenu de ces préoccupations, la question de l'UEM fut étroitement liée aux questions sociales. Au cours des années 1990, le TGWU et UNISON s'opposèrent au projet d'UEM redoutant qu'une adhésion n'aggrave les difficultés économiques de la Grande-Bretagne et ne provoque une hausse du chômage. En revanche, l'AEEU, le GMB, MSF (et le TUC) préconisèrent une adhésion à l'euro afin d'aider l'industrie manufacturière, menacée par la surévaluation de la livre. Les réserves dont ils firent dépendre leur soutien à l'UEM reflétaient ces mêmes préoccupations : outre leur insistance sur un renforcement de la dimension sociale européenne, ils ont beaucoup insisté, ces dernières années, sur la protection du secteur public.

Après avoir majoritairement soutenu une adhésion à l'euro, le mouvement syndical britannique semble aujourd'hui faire preuve de nettement moins d'enthousiasme, ce qui s'explique sans doute en partie par le silence du gouvernement sur cette question. Depuis 2003, l'euro n'a plus jamais été abordé lors des Congrès du TUC. 